\title{
Positive Solutions to Periodic Boundary Value Problems of Nonlinear Fractional Differential Equations at Resonance
}

\author{
Lei Hu \\ School of Science, Shandong Jiaotong University, Jinan 250357, China \\ Correspondence should be addressed to Lei Hu; huleimath@163.com
}

Received 9 July 2016; Accepted 23 October 2016

Academic Editor: Ahmed El-Sayed

Copyright (C) 2016 Lei Hu. This is an open access article distributed under the Creative Commons Attribution License, which permits unrestricted use, distribution, and reproduction in any medium, provided the original work is properly cited.

By Leggett-Williams norm-type theorem for coincidences due to O'Regan and Zima, we discuss the existence of positive solutions to fractional order with periodic boundary conditions at resonance. At last, an example is presented to demonstrate the main results.

\section{Introduction}

Fractional differential equations are generalizations of ordinary differential equations to an arbitrary order. It has played a significant role in many fields, such as viscoelasticity, engineering, physics, and economics; see [1-5]. During the last ten years, there are a large number of papers dealing with the existence of solutions boundary value problem for fractional differential equations; see [6-10].

Recently, there is an increasing tendency on discussion for the existence of positive solutions to boundary value problems of fractional differential equations which enriched many previous results.

In [9], Yang and Wang considered the existence of solutions for the following two-point boundary value problems for fractional differential equations:

$$
\begin{aligned}
D_{0^{+}}^{\alpha} u(t) & =f\left(t, u(t), u^{\prime}(t)\right), \quad 0<t<1, \\
u(0) & =0, \\
u^{\prime}(0) & =u^{\prime}(1),
\end{aligned}
$$

where $1<\alpha<2, D_{0^{+}}^{\alpha}$ denoting the Caputo fractional derivative. By using the Leggett-Williams norm-type theorem for coincidences due to O'Regan and Zima, the authors obtained the existence of positive solutions to the above problem.
In [10], $\mathrm{Hu}$ discussed the existence of positive solutions for a boundary value problem of fractional differential inclusions with resonant boundary conditions:

$$
\begin{aligned}
D_{0^{+}}^{\alpha} u(t) & \in f(t, u(t)), \quad 0 \leq t \leq 1, \\
u^{(i)}(0) & =0, \quad i=1,2, \ldots, n-1, \\
u(0) & =u(1),
\end{aligned}
$$

where $n-1<\alpha<n, n \geq 2, D_{0^{+}}^{\alpha}$ denotes the Caputo fractional derivative, $f:[0,1] \times \mathbb{R} \rightarrow \mathscr{F}(\mathbb{R})$, and $\mathscr{F}(\mathbb{R})$ donates the family of nonempty compact and convex subsets of $\mathbb{R}$. By using the Leggett-Williams theorem for coincidences of multivalued operators due to O'Regan and Zima, results on the existence of positive solutions were established.

Periodic boundary value problems have profound practical background and wide range of applications, such as mechanics, biology, and engineering; see [11-14]. Recently, periodic boundary value conditions of fractional order have been studied by some authors, such as [15-17].

In [16], Chen et al. studied the following periodic boundary value problem for fractional $p$-Laplacian equation:

$$
\begin{aligned}
D_{0^{+}}^{\beta} \phi_{P}\left(D_{0^{+}}^{\alpha} x(t)\right) & =f\left(t, x(t), D_{0^{+}}^{\alpha} x(t)\right), \quad t \in[0, T], \\
x(0) & =x(T), \\
D_{0^{+}}^{\alpha} x(0) & =D_{0^{+}}^{\alpha} x(T),
\end{aligned}
$$


where $0<\alpha, \beta \leq 1, D_{0^{+}}^{\alpha}$ is a Caputo fractional derivative, and $f:[0, T] \times \mathbb{R}^{2} \rightarrow \mathbb{R}$ is continuous.

In [17], Hu et al. considered the existence of solutions for the following periodic boundary value problem for fractional differential equation:

$$
\begin{aligned}
D_{0^{+}}^{\alpha} u(t) & =f\left(t, u(t), u^{\prime}(t)\right), \quad 0<t<1, \\
u(0) & =u(1), \\
u^{\prime}(0) & =u^{\prime}(1),
\end{aligned}
$$

where $1<\alpha<2, D_{0^{+}}^{\alpha}$ denotes the Caputo fractional derivative, and $f:[0,1] \times \mathbb{R}^{2} \rightarrow \mathbb{R}$ is continuous. By using the coincidence degree theory, the authors obtained the existence of solutions.

From the above works, we notice that the study of positive solutions to periodic boundary value problems of fractional order at resonance is poor. Now, the question is as follows: though the existence of solutions to (4) is obtained, how can we get the positive solutions of it? The aim of this paper is to fill the gap in the relevant literature. Our main tool is the recent Leggett-Williams norm-type theorem for coincidences due to O'Regan and Zima [18].

The rest of this paper is organized as follows. Section 2, we give some necessary notations, definitions, and lemmas. In Section 3, we obtain the existence of positive solutions of (4) by Theorem 9. Finally, an example is given to illustrate our results in Section 4.

\section{Preliminaries}

First of all, we present the necessary definitions and lemmas from fractional calculus theory. For more details, see [1].

Definition 1 (see [1]). The Riemann-Liouville fractional integral of order $\alpha>0$ of a function $f:(0, \infty) \rightarrow \mathbb{R}$ is given by

$$
I_{0^{+}}^{\alpha} f(t)=\frac{1}{\Gamma(\alpha)} \int_{0}^{t}(t-s)^{\alpha-1} f(s) d s,
$$

provided that the right-hand side is pointwise defined on $(0, \infty)$.

Definition 2 (see [1]). The Caputo fractional derivative of order $\alpha>0$ of a continuous function $f:(0, \infty) \rightarrow \mathbb{R}$ is given by

$$
D_{0^{+}}^{\alpha} f(t)=\frac{1}{\Gamma(n-\alpha)} \int_{0}^{t}(t-s)^{n-\alpha-1} f^{(n)}(s) d s,
$$

where $n-1<\alpha \leq n$, provided that the right-hand side is pointwise defined on $(0, \infty)$.

Lemma 3 (see [1]). The fractional differential equation

$$
D_{0^{+}}^{\alpha} y(t)=0
$$

has solution $y(t)=c_{0}+c_{1} t+\cdots+c_{n-1} t^{n-1}, c_{i} \in \mathbb{R}, i=0,1, \ldots, n-$ 1 , and $n=[\alpha]+1$.
Furthermore, for $y \in A C^{n}[0,1]$,

$$
\begin{aligned}
& \left(I_{0^{+}}^{\alpha} D_{0^{+}}^{\alpha} y\right)(t)=y(t)-\sum_{k=0}^{n-1} \frac{y^{(k)}(0)}{k !} t^{k}, \\
& \left(D_{0^{+}}^{\alpha} I_{0^{+}}^{\alpha} y\right)(t)=y(t) .
\end{aligned}
$$

Lemma 4 (see [1]). The relation

$$
I_{a+}^{\alpha} I_{a+}^{\beta} f(x)=I_{a+}^{\alpha+\beta} f(x)
$$

is valid in the following case: $\beta>0, \alpha+\beta>0$, and $f(x) \in$ $L_{1}(a, b)$.

In the following, let us recall some definitions on Fredholm operators and cones in Banach space (see [19]).

Let $X, Y$ be real Banach spaces. Consider a linear mapping $L: \operatorname{dom} L \subset X \rightarrow Y$ and a nonlinear operator $N: X \rightarrow Y$. Assume that

(A1) $L$ is a Fredholm operator of index zero; that is, $\operatorname{Im} L$ is closed and $\operatorname{dim} \operatorname{ker} L=\operatorname{codim} \operatorname{Im} L<\infty$.

This assumption implies that there exist continuous projections $P: X \rightarrow X$ and $Q: Y \rightarrow Y$ such that $\operatorname{Im} P=\operatorname{ker} L$ and $\operatorname{ker} Q=\operatorname{Im} L$. Moreover, since $\operatorname{dim} \operatorname{Im} Q=\operatorname{codim} \operatorname{Im} L$, there exists an isomorphism $J: \operatorname{Im} Q \rightarrow \operatorname{ker} L$. Denote by $L_{P}$ the restriction of $L$ to $\operatorname{ker} P \cap \operatorname{dom} L$. Clearly, $L_{P}$ is an isomorphism from $\operatorname{ker} P \cap \operatorname{dom} L$ to $\operatorname{Im} L$; we denote its inverse by $K_{P}: \operatorname{Im} L \rightarrow \operatorname{ker} P \cap \operatorname{dom} L$. It is known that the coincidence equation $L x=N x$ is equivalent to

$$
x=(P+J Q N) x+K_{P}(I-Q) N x .
$$

Let $C$ be a cone in $X$ such that

(i) $\mu x \in C$ for all $x \in C$ and $\mu \geq 0$,

(ii) $x,-x \in C$ implies $x=\theta$.

It is well known that $C$ induces a partial order in $X$ by

$$
x \preceq y \quad \text { iff } y-x \in C .
$$

The following property is valid for every cone in a Banach space $X$.

Lemma 5 (see [18]). Let $C$ be a cone in $X$. Then for every $u \in$ $C \backslash\{0\}$ there exists a positive number $\sigma(u)$ such that

$$
\|x+u\| \geq \sigma(u)\|u\| \quad \forall x \in C .
$$

Let $\gamma: X \rightarrow C$ be a retraction, that is, a continuous mapping such that $\gamma(x)=x$ for all $x \in C$. Set

$$
\begin{aligned}
\Psi & :=P+J Q N+K_{P}(I-Q) N, \\
\Psi_{\gamma} & :=\Psi \circ \gamma .
\end{aligned}
$$

We use the following result due to O'Regan and Zima. 
Theorem 6 (see [18]). Let $C$ be a cone in $X$ and let $\Omega_{1}, \Omega_{2}$ be open bounded subsets of $X$ with $\bar{\Omega}_{1} \subset \Omega_{2}$ and $C \cap\left(\bar{\Omega}_{2} \backslash \Omega_{1}\right) \neq \emptyset$. Assume (A1) and the following assumptions hold:

(A2) QN : $X \rightarrow Y$ is continuous and bounded and $K_{P}(I-$ Q) $N: X \rightarrow X$ is compact on every bounded subset of $X$.

(A3) $L x \neq \lambda N x$ for all $x \in C \cap \partial \Omega_{2} \cap \operatorname{Im} L$ and $\lambda \in(0,1)$.

(A4) $\gamma$ maps subsets of $\bar{\Omega}_{2}$ into bounded subsets of $C$.

(A5) $\operatorname{deg}\left\{\left.[I-(P+J Q N) \gamma]\right|_{\operatorname{ker} L}, \operatorname{ker} L \cap \Omega_{2}, 0\right\} \neq 0$.

(A6) There exists $u_{0} \in C \backslash\{0\}$ such that $\|x\| \leq \sigma\left(u_{0}\right)\|\Psi x\|$ for $x \in C\left(u_{0}\right) \cap \partial \Omega_{1}$, where $C\left(u_{0}\right)=\left\{x \in C: \mu u_{0} \preceq\right.$ $x$ for some $\mu>0\}$ and $\sigma\left(u_{0}\right)$ such that $\left\|x+u_{0}\right\| \geq$ $\sigma\left(u_{0}\right)\|x\|$ for every $x \in C$.

(A7) $(P+J Q N) \gamma\left(\partial \Omega_{2}\right) \subset C$.

(A8) $\Psi_{\gamma}\left(\bar{\Omega}_{2} \backslash \Omega_{1}\right) \subset C$.

Then the equation $L x=N x$ has a solution in the set $C \cap$ $\left(\bar{\Omega}_{2} \backslash \Omega_{1}\right)$.

\section{Main Results}

In this section, we give the existence theorems for problem (4). We write Banach space $X=Y=C[0,1]$ with the norm $\|x\|=\max _{t \in[0,1]}|x(t)|$.

Define the operator $L: \operatorname{dom} L \rightarrow X$ by

$$
L u=D_{0^{+}}^{\alpha} u,
$$

where

$$
\begin{aligned}
& \operatorname{dom} L=\left\{x \in X: D_{0^{+}}^{\alpha} u(t) \in Y, u(0)=u(1), u^{\prime}(0)\right. \\
& \left.=u^{\prime}(1)\right\} .
\end{aligned}
$$

Define the operator

$$
N: X \longrightarrow Y
$$

by

$$
N u(t)=f\left(t, u(t), u^{\prime}(t)\right) .
$$

Then problem (4) can be written by $L u=N u, u \in \operatorname{dom} L$. Let

$$
\begin{aligned}
& G(t, s) \\
& = \begin{cases}1+\frac{(t-s)^{\alpha-1}(1-s)^{2-\alpha}}{(\alpha-1) \Gamma(\alpha)}-\frac{\Gamma(\alpha)(1-s)^{\alpha}}{(\alpha-1) \Gamma(2 \alpha-1)}+\frac{(1-s)(1-\alpha t)}{(\alpha-1) \Gamma(\alpha+1)}-\frac{t^{\alpha}}{\Gamma(\alpha+1)}+\frac{\Gamma(\alpha)}{\Gamma(2 \alpha)}-\frac{1}{\alpha \Gamma(\alpha+1)}+\frac{t}{\Gamma(\alpha+1)}, & 0 \leq s<t \leq 1, \\
1-\frac{\Gamma(\alpha)(1-s)^{\alpha}}{(\alpha-1) \Gamma(2 \alpha-1)}+\frac{(1-s)(1-\alpha t)}{(\alpha-1) \Gamma(\alpha+1)}-\frac{t^{\alpha}}{\Gamma(\alpha+1)}+\frac{\Gamma(\alpha)}{\Gamma(2 \alpha)}-\frac{1}{\alpha \Gamma(\alpha+1)}+\frac{t}{\Gamma(\alpha+1)}, & 0 \leq t<s \leq 1 .\end{cases}
\end{aligned}
$$

Let $\kappa$ be a constant, which is in $(0,1)$ and satisfies

$$
\kappa G(t, s)<1 .
$$

Lemma 7. The mapping $L: \operatorname{dom} L \subset Z$ is a Fredholm operator of index zero. Furthermore, the operator $K_{P}: \operatorname{Im} L \rightarrow$ $\operatorname{dom} L \cap \operatorname{ker} P$ can be written as

where

$$
K_{P} y(t)=\int_{0}^{1} k(t, s) y(s) d s, \quad t \in[0,1],
$$

$$
k(t, s):= \begin{cases}\frac{(t-s)^{\alpha-1}}{\Gamma(\alpha)}-\frac{\Gamma(\alpha)}{\Gamma(2 \alpha-1)}(1-s)^{2 \alpha-2}+\frac{1}{\alpha \Gamma(\alpha)}(1-s)^{\alpha-1}-\frac{1}{\Gamma(\alpha)}(1-s)^{\alpha-1}, & 0 \leq s \leq t \leq 1, \\ -\frac{\Gamma(\alpha)}{\Gamma(2 \alpha-1)}(1-s)^{2 \alpha-2}+\frac{1}{\alpha \Gamma(\alpha)}(1-s)^{\alpha-1}-\frac{1}{\Gamma(\alpha)}(1-s)^{\alpha-1}, & 0 \leq t<s \leq 1 .\end{cases}
$$

$$
u(t)=c_{0}+c_{1} t, \quad c_{0}, c_{1} \in \mathbb{R} .
$$

Let $y \in \operatorname{Im} L$, so there exists a function $u(t) \in \operatorname{dom} L$ which satisfies $L \mathcal{u}(t)=y(t)$. By Lemma 3, we have

$$
u(t)=I_{0^{+}}^{\alpha} y(t)+c_{0}+c_{1} t .
$$

According to the boundary value conditions of (4), we have

$$
\operatorname{ker} L=\{c, c \in \mathbb{R}\} \cong \mathbb{R}^{1} .
$$

By $u^{\prime}(0)=u^{\prime}(1)$, we can obtain $\int_{0}^{1}(1-s)^{\alpha-2} y(s) d s=0$. On the other hand, suppose $y \in Y$ satisfies $\int_{0}^{1}(1-s)^{\alpha-2} y(s) d s=0$. 
Let $u(t)=I_{0^{+}}^{\alpha} y(t)-\left.I_{0^{+}}^{\alpha} y(t)\right|_{t=1} \cdot t$. We can easily prove $u(t) \epsilon$ dom $L$. Thus, we conclude that

$$
\operatorname{Im} L=\left\{y \in Y: \int_{0}^{1}(1-s)^{\alpha-2} y(s) d s=0\right\} .
$$

Consider the linear operator $P: X \rightarrow X$ defined by

$$
P x(t)=(\alpha-1) \int_{0}^{1}(1-s)^{\alpha-2} x(s) d s, \quad t \in[0,1] .
$$

Define the operator $Q: Y \rightarrow Y$ by

$$
Q y(t)=(\alpha-1) \int_{0}^{1}(1-s)^{\alpha-2} y(s) d s, \quad t \in[0,1] .
$$

For $u(t) \in X$, we get

$$
\begin{aligned}
P(P u) & =P\left((\alpha-1) \int_{0}^{1}(1-s)^{\alpha-2} u(s) d s\right) \\
& =(\alpha-1) \int_{0}^{1}(1-s)^{\alpha-2} u(s) d s=P u .
\end{aligned}
$$

So we have $P^{2}=P$. In the same way, $Q^{2}=Q$. We notice that $\operatorname{Im} P=\operatorname{ker} L$ and $\operatorname{ker} Q=\operatorname{Im} L$. It follows from $\operatorname{Ind} L=$ $\operatorname{dim} \operatorname{ker} L-\operatorname{codim} \operatorname{Im} L=0$ that $L$ is a Fredholm mapping of index zero.

Next, we will prove that the operator $K_{P}$ is the inverse of $\left.L\right|_{\text {dom } L \text { ker } P}$.

In fact, for $u(t) \in \operatorname{dom} L \cap \operatorname{ker} P$, we have $D_{0^{+}}^{\alpha} u(t)=y(t)$. By Lemma 3, we have $u(t)=I_{0^{+}}^{\alpha} y(t)+c_{0}+c_{1} t$. According to $u(0)=u(1)$, we get

$$
c_{1}=-I_{0^{+}}^{\alpha} y(1)
$$

By $u(t) \in \operatorname{ker} P$, that is, $(\alpha-1) \int_{0}^{1}(1-s)^{\alpha-2} u(s) d s=0$, we have

$$
c_{0}=-\Gamma(\alpha) I_{0^{+}}^{2 \alpha-1} y(1)-\frac{c_{1}}{\alpha} .
$$

Then, we have

$$
\begin{aligned}
K_{P} y(t)= & u(t)=I_{0^{+}}^{\alpha} y(t)+c_{0}+c_{1} t \\
= & I_{0^{+}}^{\alpha} y(t)-\Gamma(\alpha) I_{0^{+}}^{2 \alpha-1} y(1)+\frac{1}{\alpha} I_{0^{+}}^{\alpha} y(1) \\
& -I_{0^{+}}^{\alpha} y(1) t \\
= & \frac{1}{\Gamma(\alpha)} \int_{0}^{t}(t-s)^{\alpha-1} y(s) d s \\
& -\frac{\Gamma(\alpha)}{\Gamma(2 \alpha-1)} \int_{0}^{1}(1-s)^{2 \alpha-2} y(s) d s \\
& +\frac{1}{\alpha \Gamma(\alpha)} \int_{0}^{1}(1-s)^{\alpha-1} y(s) d s \\
& +\frac{1}{\Gamma(\alpha)} \int_{0}^{1}(1-s)^{\alpha-1} t y(s) d s \\
= & \int_{0}^{1} k(t, s) y(s) d s .
\end{aligned}
$$

It is easy to see that $L K_{P} y(t)=y(t)$. Hence, $K_{P}=$ $\left(\left.L\right|_{\operatorname{dom} L \cap \operatorname{ker} P}\right)^{-1}$. This completes the proof of Lemma 7.
Lemma 8. Assume $\Omega \subset X$ is an open bounded set such that $\operatorname{dom}(L) \cap \bar{\Omega} \neq \emptyset$; then $N$ is L-compact on $\bar{\Omega}$.

Proof. By the continuity of $f$, we can obtain that $Q N(\bar{\Omega})$ and $K_{P}(I-Q) N(\bar{\Omega})$ are bounded. Hence, for $u(t) \in \bar{\Omega}, t \in[0,1]$, there exists a positive constant $T$ such that

$$
\begin{array}{r}
|(I-Q) N u(t)| \leq T, \\
|N u(t)| \leq T .
\end{array}
$$

Thus, in the view of Arzela-Ascoli theorem, we need only to prove that $K_{P}(I-Q) N(\bar{\Omega})$ is equicontinuous.

For $0 \leq t_{1}<t_{2} \leq 1, u \in \bar{\Omega}$, we have

$$
\begin{aligned}
& \left|K_{P}(I-Q) N u\left(t_{2}\right)-K_{P}(I-Q) N u\left(t_{1}\right)\right| \\
& =\mid\left[I_{0^{+}}^{\alpha}(I-Q) N u(t)\right]_{t=t_{2}}-\Gamma(\alpha) I_{0^{+}}^{2 \alpha-1}(I-Q) \\
& \cdot N u(1)+\frac{1}{\alpha} I_{0^{+}}^{\alpha}(I-Q) N u(1)-I_{0^{+}}^{\alpha}(I-Q) \\
& \cdot N u(1) t_{2}-\left[I_{0^{+}}^{\alpha}(I-Q) N u(t)\right]_{t=t_{1}}+\Gamma(\alpha) \\
& \cdot I_{0^{+}}^{2 \alpha-1}(I-Q) N u(1)-\frac{1}{\alpha} I_{0^{+}}^{\alpha}(I-Q) N u(1) \\
& +I_{0^{+}}^{\alpha}(I-Q) N u(1) t_{1}\left|\leq \frac{1}{\Gamma(\alpha)}\right| \int_{0}^{t_{2}}\left(t_{2}-s\right)^{\alpha-1} \\
& \cdot(I-Q) N u(s) d s-\int_{0}^{t_{1}}\left(t_{1}-s\right)^{\alpha-1}(I-Q) \\
& \cdot N u(s) d s\left|+\frac{1}{\Gamma(\alpha)}\right| \int_{0}^{1}(1-s)^{\alpha-1}(I-Q) \\
& \cdot N u(s) d s|\cdot| t_{2}-t_{1} \mid \\
& \leq \frac{1}{\Gamma(\alpha)} \mid \int_{0}^{t_{1}}\left[\left(t_{2}-s\right)^{\alpha-1}-\left(t_{1}-s\right)^{\alpha-1}\right](I-Q) \\
& \cdot N u(s) d s\left|+\frac{1}{\Gamma(\alpha)}\right| \int_{t_{1}}^{t_{2}}\left(t_{2}-s\right)^{\alpha-1}(I-Q) \\
& \cdot N u(s) d s\left|+\frac{T}{\Gamma(\alpha+1)}\right| t_{2}-t_{1} \mid \leq \frac{T}{\Gamma(\alpha+1)}\left[t_{2}^{\alpha}\right. \\
& \left.-t_{1}^{\alpha}+\left(t_{2}-t_{1}\right)^{\alpha}+\left(t_{2}-t_{1}\right)\right] \text {. }
\end{aligned}
$$

Notice that $t^{\alpha}, t$ are uniformly continuous on $[0,1]$. Thus, we have that $K_{P}(I-Q) N(\bar{\Omega})$ is equicontinuous on $[0,1]$. The proof is completed.

\section{Theorem 9. Assume that}

(H1) there exist positive constants $b_{1}, b_{2}, b_{3}, c_{1}, c_{2}$, and B with

$$
B>\frac{c_{2}}{c_{1}}+\frac{3 b_{2} c_{2}}{(\alpha-1) b_{1} c_{1}}+\frac{3 b_{3}}{(\alpha-1) b_{1}} \text {. }
$$


For all $t \in[0,1]$ and $u(t) \in[0, B]$, one has

$$
\begin{aligned}
-\kappa u(t) \leq & f\left(t, u(t), u^{\prime}(t)\right) \leq-c_{1} u(t)+c_{2} \\
f\left(t, u(t), u^{\prime}(t)\right) \leq & -b_{1}\left|f\left(t, u(t), u^{\prime}(t)\right)\right|+b_{2} u(t) \\
& +b_{3}
\end{aligned}
$$

(H2) there exist $b \in(0, B), t_{0} \in[0,1], \rho \in(0,1], \delta \in$ $(0,1)$, and $q(t) \in L^{1}[0,1], q(t) \geq 0$ on $[0,1], h(x) \in$ $C\left((0, b], \mathbb{R}^{+}\right)$such that $G\left(t_{0}, s\right)>0$ and $f\left(t, u, u^{\prime}\right) \geq$ $q(t) h(u)$ for $\left(t, u, u^{\prime}\right) \in[0,1] \times(0, b] \times \mathbb{R}$. Moreover, $h(u) / u^{\rho}$ is nonincreasing on $(0, b]$ and

$$
(\alpha-1) \frac{h(b)}{b} \int_{0}^{1} G\left(t_{0}, s\right)(1-s)^{\alpha-2} q(s) d s \geq \frac{1-\delta}{\delta^{\rho}} .
$$

Then problem (4) has at least one positive solution on $[0,1]$.

Proof. According to Lemmas 7 and 8, we have that conditions (A1) and (A2) of Theorem 6 are satisfied.

Consider the cone

$$
C=\{x \in X: x(t) \geq 0, t \in[0,1]\}
$$

Let

$$
\begin{aligned}
& \Omega_{1}=\{x \in X: \delta\|x\|<|x(t)|<b, t \in[0,1]\}, \\
& \Omega_{2}=\{x \in X:\|x(t)\|<B, t \in[0,1]\} .
\end{aligned}
$$

Obviously, $\Omega_{1}$ and $\Omega_{2}$ are bounded and

$$
\bar{\Omega}_{1}=\{x \in X: \delta\|x\| \leq|x(t)| \leq b, t \in[0,1]\} \subset \Omega_{2} .
$$

Moreover, $C \cap\left(\bar{\Omega}_{2} \backslash \Omega_{1}\right) \neq \emptyset$. Let $J=I$ and $(\gamma x)(t)=|x(t)|$ for $x \in X$; then $\gamma$ is a retraction and maps subsets of $\bar{\Omega}_{2}$ into bounded subsets of $C$, which means that (A4) holds.

Next, we will show that (A3) holds. Suppose that there exist $u_{0} \in \partial \Omega_{2} \cap C \cap \operatorname{dom} L$ and $\lambda_{0} \in(0,1)$ such that $L u_{0}=$ $\lambda_{0} N u_{0}$; that is, $D_{0^{+}}^{\alpha} u_{0}(t)=\lambda_{0} f\left(t, u_{0}(t), u_{0}^{\prime}(t)\right), t \in[0,1]$. In view of $(\mathrm{H} 1)$, we get

$$
\begin{aligned}
D_{0^{+}}^{\alpha} u_{0}(t)= & \lambda_{0} f\left(t, u_{0}(t), u_{0}^{\prime}(t)\right) \\
\leq & -\lambda_{0} b_{1}\left|f\left(t, u_{0}(t), u_{0}^{\prime}(t)\right)\right|+\lambda_{0} b_{2} u_{0}(t) \\
& +\lambda_{0} b_{3} \\
= & -b_{1}\left|\lambda_{0} f\left(t, u_{0}(t), u_{0}^{\prime}(t)\right)\right|+\lambda_{0} b_{2} u_{0}(t) \\
& +\lambda_{0} b_{3} \\
= & -b_{1}\left|D_{0^{+}}^{\alpha} u_{0}(t)\right|+\lambda_{0} b_{2} u_{0}(t)+\lambda_{0} b_{3} \\
\leq & -b_{1}\left|D_{0^{+}}^{\alpha} u_{0}(t)\right|+b_{2} u_{0}(t)+b_{3}, \\
D_{0^{+}}^{\alpha} u_{0}(t)= & \lambda_{0} f\left(t, u_{0}(t), u_{0}^{\prime}(t)\right) \\
\leq & -\lambda_{0} c_{1} u_{0}(t)+\lambda_{0} c_{2} .
\end{aligned}
$$

In view of $D_{0^{+}}^{\alpha} u_{0}(t)=\lambda_{0} f\left(t, u_{0}(t)\right) \in \operatorname{Im} L$, from the definition of $\operatorname{Im} L$ and (41), we obtain

$$
\begin{aligned}
0 & =\int_{0}^{1}(1-s)^{\alpha-2} D_{0^{+}}^{\alpha} u_{0}(s) d s \\
& \leq \int_{0}^{1}(1-s)^{\alpha-2}\left(-\lambda_{0} c_{1} u_{0}(s)+\lambda_{0} c_{2}\right) d s
\end{aligned}
$$

which gives

$$
\int_{0}^{1}(1-s)^{\alpha-2} u_{0}(s) d s \leq \frac{c_{2}}{(\alpha-1) c_{1}} .
$$

From (40), we have

$$
\begin{aligned}
0= & \int_{0}^{1}(1-s)^{\alpha-2} D_{0^{+}}^{\alpha} u_{0}(s) d s \\
\leq & \int_{0}^{1}(1-s)^{\alpha-2}\left[-b_{1}\left|D_{0^{+}}^{\alpha} u_{0}(t)\right|+b_{2} u_{0}(t)+b_{3}\right] d s \\
= & -b_{1} \int_{0}^{1}(1-s)^{\alpha-2}\left|D_{0^{+}}^{\alpha} u_{0}(s)\right| d s \\
& +b_{2} \int_{0}^{1}(1-s)^{\alpha-2} u_{0}(s) d s+\frac{b_{3}}{\alpha-1} .
\end{aligned}
$$

By (43), we have

$$
\begin{aligned}
& \int_{0}^{1}(1-s)^{\alpha-2}\left|D_{0^{+}}^{\alpha} u_{0}(s)\right| d s \\
& \quad \leq \frac{b_{2}}{b_{1}} \int_{0}^{1}(1-s)^{\alpha-2} u_{0}(s) d s+\frac{b_{3}}{(\alpha-1) b_{1}} \\
& \quad \leq \frac{b_{2} c_{2}}{(\alpha-1) b_{1} c_{1}}+\frac{b_{3}}{(\alpha-1) b_{1}} .
\end{aligned}
$$

According to the function expression of $k(t, s)$, it is easy to see that $|k(t, s)| \leq 3(1-s)^{\alpha-2}, s, t \in[0,1]$. From (43) and the equation $u_{0}=(I-P) u_{0}+P u_{0}=K_{P} L(I-P) u_{0}+P u_{0}=$ $P u_{0}+K_{P} L u_{0}$, we can get

$$
\begin{aligned}
u_{0}= & (\alpha-1) \int_{0}^{1}(1-s)^{\alpha-2} u_{0}(s) d s \\
& +\int_{0}^{1} k(t, s) D_{0^{+}}^{\alpha} u_{0}(s) d s \\
\leq & (\alpha-1) \cdot \frac{c_{2}}{(\alpha-1) c_{1}}+\int_{0}^{1}|k(t, s)| \cdot\left|D_{0^{+}}^{\alpha} u_{0}(s)\right| d s \\
= & \frac{c_{2}}{c_{1}}+\int_{0}^{1} \frac{|k(t, s)|}{(1-s)^{\alpha-2}} \cdot(1-s)^{\alpha-2}\left|D_{0^{+}}^{\alpha} u_{0}(s)\right| d s \\
\leq & \frac{c_{2}}{c_{1}}+3 \int_{0}^{1}(1-s)^{\alpha-2}\left|D_{0^{+}}^{\alpha} u_{0}(s)\right| d s \\
\leq & \frac{c_{2}}{c_{1}}+\frac{3 b_{2} c_{2}}{(\alpha-1) b_{1} c_{1}}+\frac{3 b_{3}}{(\alpha-1) b_{1}} .
\end{aligned}
$$


Then, we have

$$
B=\left\|u_{0}\right\| \leq \frac{c_{2}}{c_{1}}+\frac{3 b_{2} c_{2}}{(\alpha-1) b_{1} c_{1}}+\frac{3 b_{3}}{(\alpha-1) b_{1}},
$$

which contradicts (H1). Hence (A3) holds.

To prove (A5), consider $u(t) \in \operatorname{ker} L \cap \bar{\Omega}_{2}$; then $u(t) \equiv c$. For $c \in[-B, B]$ and $\lambda \in[0,1]$, we have

$$
\begin{aligned}
& H(c, \lambda)=[I-\lambda(P+J Q N) \gamma] c \\
& =c-\lambda(\alpha-1) \int_{0}^{1}(1-s)^{\alpha-2}|c| d s \\
& \quad-\lambda(\alpha-1) \int_{0}^{1}(1-s)^{\alpha-2} f(s,|c|, 0) d s \\
& =c-\lambda|c|-\lambda(\alpha-1) \int_{0}^{1}(1-s)^{\alpha-2} f(s,|c|, 0) d s \\
& =c-\lambda(\alpha-1) \int_{0}^{1}(1-s)^{\alpha-2}[f(s,|c|, 0)+|c|] d s .
\end{aligned}
$$

By use of proof by contradiction, it is easy to show that $H(c, \lambda)=0$ implies $c \geq 0$. Suppose $H(B, \lambda)=0$ for some $\lambda \in(0,1]$; then we have

$$
0=B-\lambda B-\lambda(\alpha-1) \int_{0}^{1}(1-s)^{\alpha-2} f(s, B) d s
$$

According to (H1), we have

$$
\begin{aligned}
0 & \leq B(1-\lambda)=\lambda(\alpha-1) \int_{0}^{1}(1-s)^{\alpha-2} f(s, B, 0) d s \\
& \leq \lambda\left(-c_{1} B+c_{2}\right)<0,
\end{aligned}
$$

which is a contradiction. In addition, if $\lambda=0$, then $B=0$, which is impossible. As a result, for $x \in \operatorname{ker} L \cap \partial \Omega_{2}$ and $\lambda \epsilon$ $[0,1]$, we have $H(x, \lambda) \neq 0$. Thus,

$$
\begin{aligned}
\operatorname{deg} & \left\{[I-(P+J Q N) \gamma]_{\operatorname{ker} L}, \operatorname{ker} L \cap \Omega_{2}, 0\right\} \\
& =\operatorname{deg}\left\{H(\cdot, 1), \operatorname{ker} L \cap \Omega_{2}, 0\right\} \\
& =\operatorname{deg}\left\{H(\cdot, 0), \operatorname{ker} L \cap \Omega_{2}, 0\right\} \\
& =\operatorname{deg}\left\{I, \operatorname{ker} L \cap \Omega_{2}, 0\right\}=1 \neq 0 .
\end{aligned}
$$

So (A5) holds.

Next, we prove (A6). Let $u_{0}(t) \equiv 1, t \in[0,1]$; then $u_{0} \in$ $C \backslash\{0\}, C\left(u_{0}\right)=\{x \in C: x(t)>0, t \in[0,1]\}$. We take $\sigma\left(u_{0}\right)=1$. Let $x \in C\left(u_{0}\right) \cap \partial \Omega_{1}$; then $0<\|x\| \leq b$ and $x(t) \geq \delta\|x\|$ on $[0,1]$.
By (H2), for every $x \in C\left(u_{0}\right) \cap \partial \Omega_{1}$, we have

$$
\begin{aligned}
& (\Psi) x\left(t_{0}\right)=\left[\left(P+J Q N+K_{P}(I-Q) N\right) x(t)\right]_{t=t_{0}} \\
& =(\alpha-1) \int_{0}^{1}(1-s)^{\alpha-2} x(s) d s+(\alpha-1) \\
& \cdot \int_{0}^{1} G\left(t_{0}, s\right)(1-s)^{\alpha-2} f\left(s, x(s), x^{\prime}(s)\right) d s \\
& \geq(\alpha-1) \int_{0}^{1}(1-s)^{\alpha-2} \delta\|x\| d s+(\alpha-1) \\
& \cdot \int_{0}^{1} G\left(t_{0}, s\right)(1-s)^{\alpha-2} f\left(s, x(s), x^{\prime}(s)\right) d s \\
& \geq \delta\|x\|+(\alpha-1) \\
& \cdot \int_{0}^{1} G\left(t_{0}, s\right)(1-s)^{\alpha-2} q(s) h(x(s)) d s=\delta\|x\| \\
& +(\alpha-1) \\
& \cdot \int_{0}^{1} G\left(t_{0}, s\right)(1-s)^{\alpha-2} q(s) \cdot \frac{h(x(s))}{x^{\rho}(s)} x^{\rho}(s) d s \\
& \geq \delta\|x\|+(\alpha-1) \\
& \cdot \int_{0}^{1} G\left(t_{0}, s\right)(1-s)^{\alpha-2} q(s) \cdot \frac{h(x(s))}{x^{\rho}(s)} \cdot \delta^{\rho}\|x\|^{\rho} d s \\
& \geq \delta\|x\|+\delta^{\rho}\|x\|^{\rho}(\alpha-1) \\
& \cdot \int_{0}^{1} G\left(t_{0}, s\right)(1-s)^{\alpha-2} q(s) \cdot \frac{h(b)}{b^{\rho}} d s=\delta\|x\| \\
& +\delta^{\rho}\|x\| \cdot \frac{b^{1-\rho}}{\|x\|^{1-\rho}}(\alpha-1) \\
& \cdot \int_{0}^{1} G\left(t_{0}, s\right)(1-s)^{\alpha-2} q(s) \frac{h(b)}{b} d s \geq \delta\|x\| \\
& +\delta^{\rho}\|x\| \cdot(\alpha-1) \\
& \cdot \int_{0}^{1} G\left(t_{0}, s\right)(1-s)^{\alpha-2} q(s) \frac{h(b)}{b} d s \geq \delta\|x\| \\
& +\delta^{\rho}\|x\| \cdot \frac{1-\delta}{\delta^{\rho}}=\|x\| .
\end{aligned}
$$

Thus, for all $x \in C\left(u_{0}\right) \cap \partial \Omega_{1}$, we have $\|x\| \leq \sigma\left(u_{0}\right)\|\Psi x\|$; that is, (A6) holds.

For $x \in \partial \Omega_{2}$, by (H2) and (19), we have

$$
\begin{aligned}
& {[(P+J Q N) \circ \gamma] x(t)=P(|x(t)|)+J Q N(|x(t)|)} \\
& =(\alpha-1) \int_{0}^{1}(1-s)^{\alpha-2}|x(s)| d s \\
& \quad+(\alpha-1) \int_{0}^{1}(1-s)^{\alpha-2} f\left(s,|x(s)|,(|x(s)|)^{\prime}\right) d s \\
& \geq(\alpha-1) \int_{0}^{1}(1-s)^{\alpha-2}(1-\kappa)|x(s)| d s \geq 0 .
\end{aligned}
$$

Thus, $[(P+J Q N) \circ \gamma] x(t) \subset C$ for $x \in \partial \Omega_{2}$. Then (A7) holds. 
Next, we prove (A8). For $x(t) \in \bar{\Omega}_{2} \backslash \Omega_{1}$, by (H2), we have

$$
\begin{aligned}
& \Psi_{\gamma} x(t)=\left[\left(P+J Q N+K_{P}(I-Q) N\right) \circ \gamma\right] x(t) \\
& \quad=\left(P+J Q N+K_{P}(I-Q) N\right)|x(t)|=P(|x(t)|) \\
& +\left(J Q N+K_{P}(I-Q) N\right)|x(t)|=(\alpha-1) \\
& \cdot \int_{0}^{1}(1-s)^{\alpha-2}|x(s)| d s+(\alpha-1) \\
& \cdot \int_{0}^{1}(1-s)^{\alpha-2} G(t, s) f\left(s,|x(s)|,(|x(s)|)^{\prime}\right) d s \\
& >(\alpha-1) \int_{0}^{1}(1-s)^{\alpha-2}|x(s)| d s+(\alpha-1) \\
& \cdot \int_{0}^{1}(1-s)^{\alpha-2} G(t, s)(-\kappa|x(s)|) d s>(\alpha-1) \\
& \cdot \int_{0}^{1}(1-s)^{\alpha-2}|x(s)|(1-\kappa G(t, s)) d s \geq 0 .
\end{aligned}
$$

Hence, $\Psi_{\gamma}\left(\bar{\Omega}_{2} \backslash \Omega_{1}\right) \subset C$; that is, (A8) holds.
Hence, applying Theorem 6, BVP (4) has a positive solution $u^{*}(t)$ on $[0,1]$ with $b \leq\left\|u^{*}(t)\right\| \leq B$. This completes the proof.

\section{Example}

To illustrate how our main result can be used in practice, we present here an example.

Let us consider the following fractional differential equation at resonance:

$$
\begin{aligned}
D_{0^{+}}^{1.5} u(t) & =f\left(t, u(t), u^{\prime}(t)\right), \quad 0<t<1, \\
u(0) & =u(1), \\
u^{\prime}(0) & =u^{\prime}(1),
\end{aligned}
$$

where

$$
\begin{aligned}
f\left(t, u(t), u^{\prime}(t)\right) \\
=\frac{1}{400}\left(1+t-t^{2}\right)\left(u^{2}(t)-8 u(t)+12\right) u(t) \\
\quad+\frac{1}{30} e^{-\left|u^{\prime}(t)\right|} .
\end{aligned}
$$

Corresponding to BVP (4), we have that $\alpha=1.5$ and

$$
\begin{aligned}
& G(t, s) \\
& = \begin{cases}1+\frac{(t-s)^{0.5}(1-s)^{0.5}}{0.5 \Gamma(1.5)}-\frac{\Gamma(1.5)(1-s)^{1.5}}{0.5 \Gamma(2)}+\frac{(1-s)(1-1.5 t)}{0.5 \Gamma(2.5)}-\frac{t^{1.5}}{\Gamma(2.5)}+\frac{\Gamma(1.5)}{\Gamma(3)}-\frac{1}{1.5 \Gamma(2.5)}+\frac{t}{\Gamma(2.5)}, & 0 \leq s<t \leq 1, \\
1-\frac{\Gamma(1.5)(1-s)^{1.5}}{0.5 \Gamma(2)}+\frac{(1-s)(1-1.5 t)}{0.5 \Gamma(2.5)}-\frac{t^{1.5}}{\Gamma(2.5)}+\frac{\Gamma(1.5)}{\Gamma(3)}-\frac{1}{1.5 \Gamma(2.5)}+\frac{t}{\Gamma(2.5)}, & 0 \leq t<s \leq 1 .\end{cases}
\end{aligned}
$$

By simple calculation, we can get that if $t \in[0,1]$ and $u \in$ $[0,1]$, one has

$$
\begin{aligned}
-\frac{1}{15} u(t) \leq & f\left(t, u(t), u^{\prime}(t)\right) \\
\leq & \frac{1}{20} u(t)+\frac{1}{10}, \\
f\left(t, u(t), u^{\prime}(t)\right) \leq & -39\left|f\left(t, u(t), u^{\prime}(t)\right)\right|+\frac{1}{2} u(t) \\
& +\frac{8}{5} .
\end{aligned}
$$

So, we can choose $\kappa=1 / 15, B=1, c_{1}=1 / 20, c_{2}=1 / 10$, $b_{1}=39, b_{2}=1 / 2$, and $b_{3}=8 / 5$. Furthermore, we can verify

$$
B>\frac{c_{2}}{c_{1}}+\frac{3 b_{2} c_{2}}{(\alpha-1) b_{1} c_{1}}+\frac{3 b_{3}}{(\alpha-1) b_{1}} .
$$

So, (H1) is satisfied.
Taking $\rho=1, h(u)=(1 / 20) u$, and $q(t)=(33 / 80)(1+t-$ $t^{2}$ ), we have

$$
\begin{aligned}
f\left(t, u(t), u^{\prime}(t)\right) & \geq q(t) h(u) \\
= & \frac{1}{20} u(t) \cdot \frac{33}{85}\left(1+t-t^{2}\right), \\
& u(t) \in[0,1], t \in[0,1] .
\end{aligned}
$$

Let $t_{0}=0$; then

$$
\begin{aligned}
G(0, s)= & 1-2 \Gamma(1.5)(1-s)^{1.5}+\frac{2}{\Gamma(2.5)}(1-s) \\
& +\frac{\Gamma(1.5)}{2}-\frac{2}{3 \Gamma(2.5)}>0 .
\end{aligned}
$$

Moreover, take $b=1 / 2 \in[0,1]$, and we have that

$$
\begin{aligned}
& (\alpha-1) \frac{h(b)}{b} \int_{0}^{1} G\left(t_{0}, s\right)(1-s)^{\alpha-2} q(s) d s \approx 0.98 \\
& >\frac{1-\delta}{\delta^{\rho}}=0.97,
\end{aligned}
$$




$$
\frac{h(u)}{u^{\rho}}=\frac{(1 / 20) u(t)}{u^{\rho}(t)}=\frac{1}{20}
$$

is nonincreasing on $(0,1 / 2] \delta=0.9995$. So, conditions (H1)(H2) of Theorem 9 are satisfied; then BVP (55) has a positive solution on $[0,1]$.

\section{Competing Interests}

The author declares that he has no competing interests.

\section{Authors' Contributions}

The author contributed to the writing of this paper. The author read and approved the final manuscript.

\section{Acknowledgments}

Research was supported by the Science Foundation of Shandong Jiaotong University (Z201429).

\section{References}

[1] A. A. Kilbas, H. M. Srivastava, and J. Trujillo, Theory and Applications of Fractional Differential Equations, vol. 204, Elsevier, Amsterdam, The Netherlands, 2006.

[2] I. Podlubny, Fractional Differential Equations, vol. 198 of Mathematics in Science and Engineering, Academic Press, San Diego, Calif, USA, 1999.

[3] S. Ladaci, J. J. Loiseau, and A. Charef, "Fractional order adaptive high-gain controllers for a class of linear systems," Communications in Nonlinear Science and Numerical Simulation, vol. 13, no. 4, pp. 707-714, 2008.

[4] V. Gafiychuk, B. Datsko, and V. Meleshko, "Mathematical modeling of time fractional reaction-diffusion systems," Journal of Computational and Applied Mathematics, vol. 220, no. 1-2, pp. 215-225, 2008.

[5] F. C. Meral, T. J. Royston, and R. Magin, "Fractional calculus in viscoelasticity: an experimental study," Communications in Nonlinear Science and Numerical Simulation, vol. 15, no. 4, pp. 939-945, 2010.

[6] Z. Bai and H. Lü, "Positive solutions for boundary value problem of nonlinear fractional differential equation," Journal of Mathematical Analysis and Applications, vol. 311, no. 2, pp. 495-505, 2005.

[7] S. Zhang, "Existence of solutions for two-point boundary-value problems with singular differential equations of variable order," Electronic Journal of Differential Equations, vol. 2013, no. 245, pp. 1-16, 2013.

[8] J. Henderson and R. Luca, "Existence of positive solutions for a system of semipositone fractional boundary value problems," Electronic Journal of Qualitative Theory of Differential Equations, vol. 2016, pp. 1-22, 2016.

[9] A. Yang and H. Wang, "Positive solutions of two-point boundary value problems of nonlinear fractional differential equation at resonance," Electronic Journal of Qualitative Theory of Differential Equations, no. 71, pp. 1-15, 2011.

[10] $\mathrm{L}$. Hu, "On the existence of positive solutions for fractional differential inclusions at resonance," SpringerPlus, vol. 5, no. 1, pp. 1-12, 2016.
[11] E. Bravyi, "On solvability of periodic boundary value problems for second order linear functional differential equations," Electronic Journal of Qualitative Theory of Differential Equations, vol. 2016, pp. 1-18, 2016.

[12] M. Belmekki, J. J. Nieto, and R. Rodriguez-López, "Existence of periodic solution for a nonlinear fractional differential equation," Boundary Value Problems, vol. 2009, Article ID 324561, 18 pages, 2009.

[13] H. Jin and W. Liu, "On the periodic boundary value problem for Duffing type fractional differential equation with $p$-Laplacian operator," Boundary Value Problems, vol. 2015, article 144, pp. 1-11, 2015.

[14] M. Zima and P. Drygaś, "Existence of positive solutions for a kind of periodic boundary value problem at resonance," Boundary Value Problems, vol. 2013, article 19, 10 pages, 2013.

[15] Z. Wei, W. Dong, and J. Che, "Periodic boundary value problems for fractional differential equations involving a RiemannLiouville fractional derivative," Nonlinear Analysis: Theory, Methods \& Applications, vol. 73, no. 10, pp. 3232-3238, 2010.

[16] T. Chen, W. Liu, and J. Liu, "Solvability of periodic boundary value problem for fractional $p$-Laplacian equation," Applied Mathematics and Computation, vol. 244, pp. 422-431, 2014.

[17] Z. Hu, W. Liu, and W. Rui, "Periodic boundary value problem for fractional differential equation," International Journal of Mathematics, vol. 23, no. 10, Article ID 1250100, pp. 1-11, 2012.

[18] D. O'Regan and M. Zima, "Leggett-Williams norm-type theorems for coincidences," Archiv der Mathematik, vol. 87, no. 3, pp. 233-244, 2006.

[19] J. Mawhin, Topological Degree Methods in Nonlinear Boundary Value Problems, vol. 40 of NSFCBMS Regional Conference Series in Mathematics, American Mathematical Society, Providence, RI, USA, 1979. 


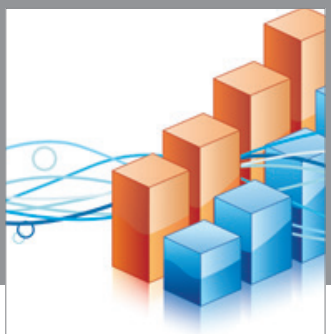

Advances in

Operations Research

vatem alat4

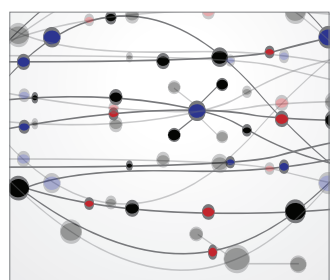

\section{The Scientific} World Journal
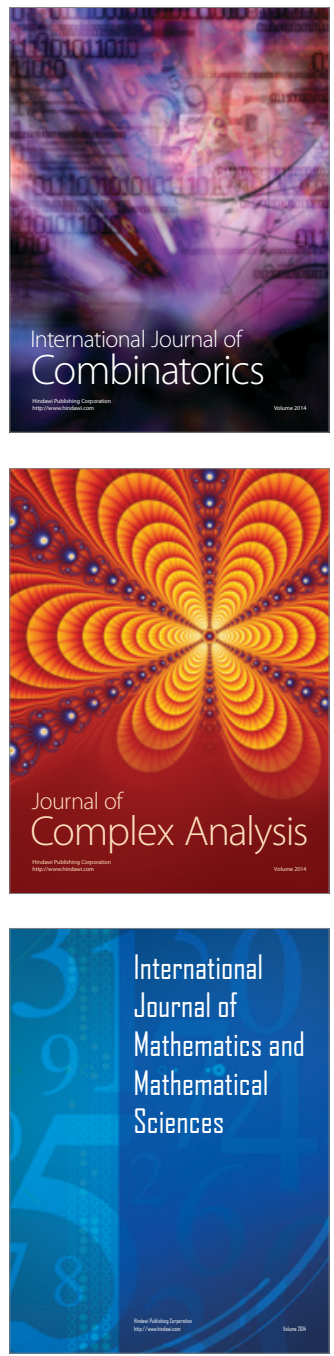
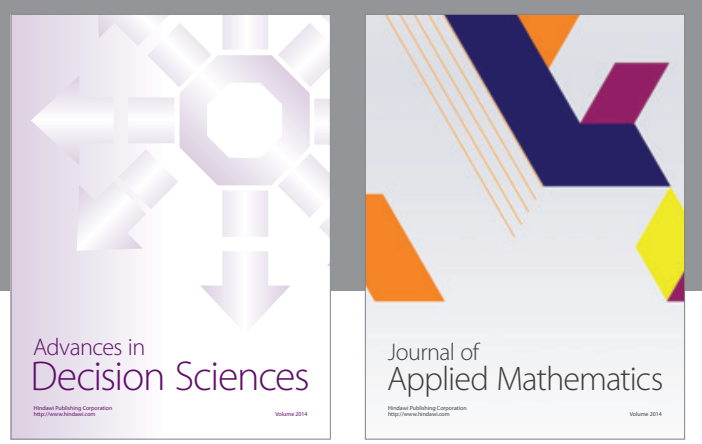

Algebra

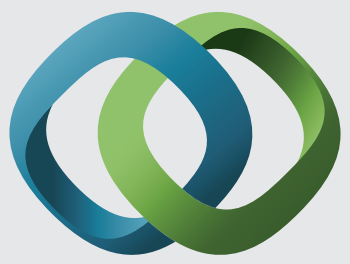

\section{Hindawi}

Submit your manuscripts at

http://www.hindawi.com
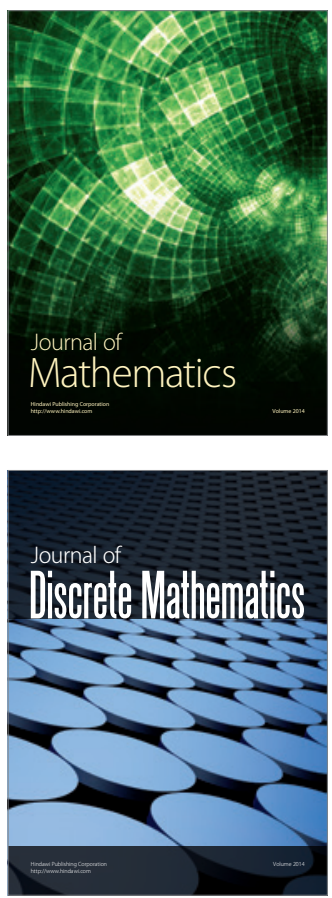

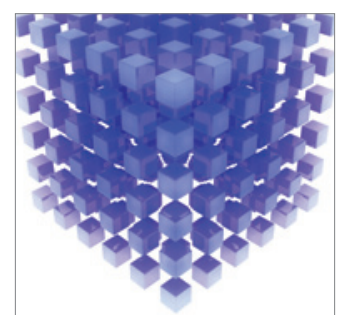

Mathematical Problems in Engineering
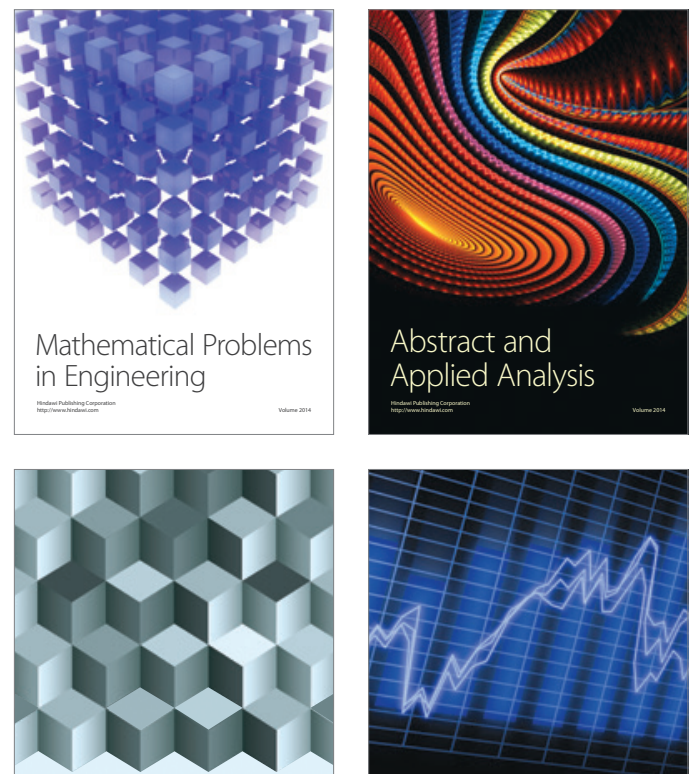

Journal of

Function Spaces

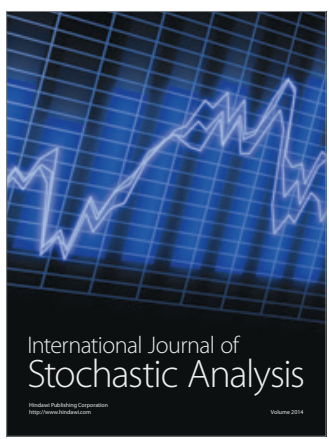

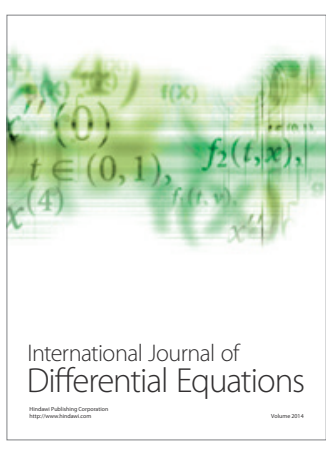
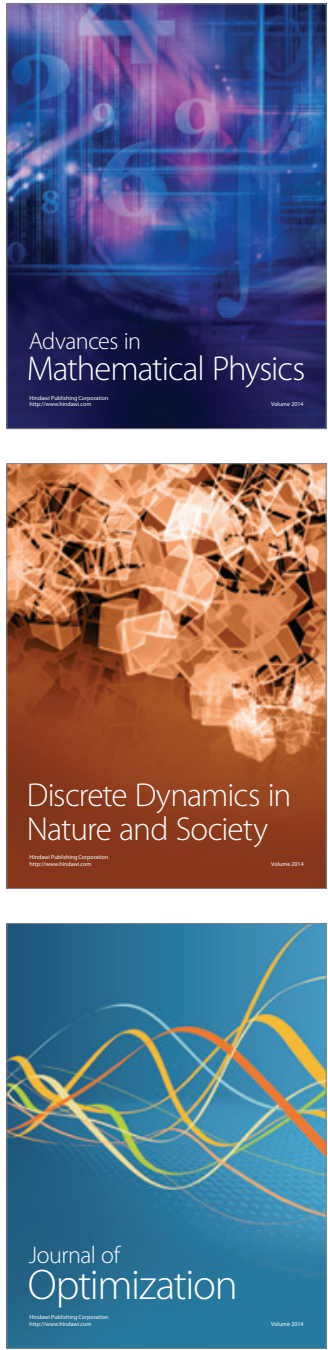